\title{
Teaching fiber-optic communications in engineering technology programs by virtual collaboration with industry
}

\section{Djafar Mynbaev}

Djafar K. Mynbaev, "Teaching fiber-optic communications in engineering technology programs by virtual collaboration with industry," Proc. SPIE 9663, Eighth International Topical Meeting on Education and Training in Optics and Photonics, 96630G (6 October 2003); doi: 10.1117/12.2207347

SPIE Event: Eighth International Topical Meeting on Education and Training in Optics and Photonics, 2003, Tucson, Arizona, United States 


\title{
Teaching fiber-optic communications in engineering technology programs by virtual collaboration with industry
}

\author{
Djafar K. Mynbaev \\ New York City College of Technology of the City University of New York, 300 Jay Street, V-733, Brooklyn, New York 11201 \\ dmynbaev@citytech.cuny.edu
}

\begin{abstract}
A fiber-optic communications course requires a deep understanding of the physical processes of the components and systems. Unfortunately, many students in engineering technology programs lack the scientific background for such a course. Another challenge is that these students need to be trained as maintenance and control personnel. To resolve these problems, we focus our teaching on the use of corporate technical documentation.

(C) 2003 Optical Society of America

OCIS codes: (000.2060) Education; (060.2330) fiber optics communications
\end{abstract}

\section{Fiber-optic communications courses and engineering technology programs}

Fiber-optic communications has become the linchpin of the modern telecommunication industry. In fact, more than $98 \%$ of U.S. domestic traffic uses fiber cable. This is why more and more engineering technology schools offer a variety of courses related to fiber-optic communications. Since fiber-optic communications technology includes both component and networking aspects, such courses have to lean on knowledge that students have gathered in physics, mathematics, electronics, telecommunications, and other engineering and science courses. However, analysis of fiber-optic technical documentation relies not only on broad education but also on specific knowledge acquired through the study of fiber-optic technology.

Another important feature of fiber-optic communications technology is that it changes very rapidly. Every day brings new developments in components, networking, and even business conditions. These developments can be as simple as some improvements in the characteristics of a component, a new design in the architecture of a specific network, or an increase in production by a specific company. But these developments can also be the invention of new devices, such as erbium-doped or Raman fiber amplifiers; new transmission technology, such as wavelengthdivision multiplexing; or a new approach to networking, such as the all-optical network. From the business standpoint, one has to read the professional journals every day to follow mergers, acquisitions, and spinoffs. Hundreds of start-up companies appear and disappear and well-established companies change their names and profiles. Thus, teaching a fiber-optic communications course requires a permanent updating of the lecture material and laboratory exercises, which means bringing breaking industry developments into the classroom and laboratory.

Therefore, teaching a fiber-optic communication course presents a challenge. On the one hand, it should provide deep academic knowledge about the subject. On the other hand, the subject itself changes so rapidly that classroom-acquired knowledge becomes outdated before students reach the workplace. The solution lies in finding the right balance in providing fundamental knowledge and teaching the current technology. This is where close collaboration with industry has to come into the play.

When teaching in an engineering technology program, the instructor must address two critical issues: The first is the background in the basic sciences of students in engineering- technology programs. Typically, their mathematical education doesn't include courses beyond Calculus II, and their education in the physical sciences doesn't exceed two courses in non-calculus physics. The problem is the scientific descriptions of processes, devices, and systems that have to be learned require a thorough grounding in the fundamentals of mathematics, physics, and other disciplines. A fiber-optic communications course is particularly challenging because it relies heavily on a deep understanding of the physical processes that control the behavior of the components and systems constituting this technology. Optical fiber itself, light sources, photodiodes, and all the passive and active components of fiber-optic networks demand an in-depth knowledge of classical optics, semiconductor materials, micromechanical and microoptical processes, and many aspects of modern science.

The second point is the nature of the future work and potential responsibilities of the graduates of engineering technology programs. They are trained to work as technologists, which means their major 
responsibilities lie mostly in maintenance and control; however, the tendency is to involve them in design, development, and research. Thus, their academic training has to provide them with a deep knowledge of technology coupled with a sound general education. This knowledge entails a complete understanding of corporate technical documentation, particularly, company specifications sheets. Thus, again, engineering-technology education must rely heavily on exposing students to industry practice, which can only be achieved by collaboration with industry.

There are many forms of collaboration between academe and industry most of them requiring the physical presence of students at company workplaces. Unfortunately, this requirement is often difficult to meet and the effectiveness of the student at the work site is always questionable. This is why we propose a virtual collaboration with industry without the need for the physical presence of the student at the workplace. This type of collaboration introduces industry practice in the academic environment. To implement this type of collaboration, we bring two key elements of industry practice to the classroom. First, we simulate a realistic working environment in our teaching. Secondly, we use corporate technical documentation, especially the manufacturers' specifications sheets, as a teaching material.

The following two examples demonstrate our approach to virtual collaboration with industry in the laboratory and classroom in our fiber-optic communications course at the senior level in our undergraduate telecommunications-technology program.

\section{Measuring attenuation of a multimode fiber (MMF) in the laboratory}

Here we want to measure attenuation of an MMF. The main point is to investigate how the main performance characteristic of an optical fiber - attenuation - depends on the length of a fiber-optic communications link.

Attenuation is the first characteristic of any transmission medium that a telecommunications engineer will be looking at when choosing a link. In fact, optical fiber found application as a practical transmission medium only when its attenuation was reduced to the current number, about $0.2 \mathrm{~dB} / \mathrm{km}$. Thus, understanding attenuation is a critical point in studying the properties of an optical fiber. Attenuation in MMFs is particularly interesting because, in contrast to attenuation in singlemode fiber (SMF), this attenuation changes with a change of the fiber length, which is the only proof of the existence of modes in optical fiber.

The main feature of our laboratory exercises is that we try to conduct them the same way engineers carry their projects out in the workplace.

Each experiment is designed as a realistic working assignment, which means that only the goal to be achieved through the experiment and some preliminary information are all the information provided. The student is given no detailed step-by-step instructions. Using their knowledge of the topic obtained in the classroom and from the textbook [1], the student has to devise by himself or herself the arrangement and the procedure of measurement and realize what data are to be gathered and how that data must be processed. The student has to build the setup of the experiment and the necessary circuitry and perform the measurements.

As an example of the implementation of this approach, let's consider the following laboratory exercise: The underlined text shows what the students receive as a part of their laboratory manual. This portion of the manual represents the students' assignment. Text in italics shows how the assignment should be carried out, which includes the reasoning and practical steps that have to be developed.

\section{Experiment 1. Measuring attenuation in optical fibers}

Equipment: IF-97 ED, WL-850 calibrated light source, cables of SuperEska plastic optical fiber, cables of silica optical fiber by Siecor, a trainer board, resistors, and a power meter

\section{Assignment: Collect data to evaluate attenuation of multimode optical fiber}

\section{Implementation:}

First, students become familiar with all equipment provided. They learn how to use a trainer board, a power meter, and a calibrated light source. They become familiar with the characteristics of all the devices by studying the technical documentation (data sheets), which are part of the laboratory manual ${ }^{2}$. For example, they find out that a power meter can measure over a certain range of wavelength, namely, 650 $\mathrm{nm}, 850 \mathrm{~nm}$, and $910 \mathrm{~nm}$. Secondly, it is expected that the students will develop the following reasoning:

1. Attenuation is given by [1]

$$
A(d B / k m)=- \text { Loss }(d B) / \text { Fiber length }(\mathrm{km}),
$$


where Loss $(\mathrm{dB})=10 \log P_{\text {out }}(\mathrm{mW}) / P_{\text {in }}(\mathrm{mW})=P_{\text {out }}(\mathrm{dBm})-P_{\text {in }}(\mathrm{dBm})$

Therefore, to obtain the attenuation, we need to measure output and input power either in $\mathrm{mW}$ or in $d B m$.

2. Input power, $P_{i n}$, is not the power radiated by a light source, but it is the power launched into an optical fiber. Therefore, if we measure the light power emerging from a short piece of optical fiber, we will obtain the desired quantity, $P_{i n}$. This is because the losses introduced by this short piece are negligible.

3. Output power, $P_{\text {out }}$, is the light power emerging from the optical fiber under test. Thus, to obtain attenuation, we need to measure $P_{i n}, P_{\text {out }}$, and the fiber length.

4. It follows from the definition of attenuation that $A(\mathrm{~dB} / \mathrm{km})$ should be independent of fiber length. However, this is not true for multimode fibers. To investigate this phenomenon, we need to take measurements of attenuation with fiber cables of various lengths.

5. Thus, we arrive at the following procedure for the experiment [2]:

Choose a light source after looking at the specifications sheets of a light source and a specific optical fiber.

Measure the light power emerging from a short piece of optical fiber.

Measure the light power emerging from multimode optical fiber cables of different lengths.

Record the length of each fiber cable.

Each student has to write an individual laboratory report. This paper is considered as a technical report that the student would have to submit to his or her potential supervisor after completing an assignment in the workplace. Writing this report requires the students to express their thoughts in a professional manner, with an emphasis on appropriate grammar and style.

The laboratory manual includes guidelines and report requirements. These requirements emphasize that the report must provide enough information to enable anyone to reproduce the reported measurements. A theoretical discussion must be included in the report and it must contain the qualitative predictions of the expected results of the measurements. Another important consideration is analysis of the results. For example, the student must compare the results obtained with the theoretical predictions and with the characteristics given in the manufacturer's data sheet. In our example, that is, Experiment 1, the values of attenuation computed from the measured losses must be compared with the attenuation given in manufacturer's data sheet attached to the laboratory manual. The analysis must include a discussion of any discrepancies among theory predictions, the manufacturer's data, and the results of the actual measurements.

What follows is the reasoning that we expect the students will express in the theoretical section of their laboratory report:

Attenuation was introduced by the fiber-optics communications industry in order to obtain a real measure of a fiber's quality. Indeed, absorption, scattering, and bending cause the loss of optical power inside an optical fiber. Therefore, the longer the fiber, the greater the absorption, scattering, and bending losses are while a light beam propagates through the fiber. In other words, the longer the fiber, the greater the losses. Thus, if we divide the value of the fiber's losses by length, we obtain the characteristic that really describes the loss property of a fiber, regardless of its length. Attenuation, as follows from the above consideration, should be independent of fiber length; that is, it should be constant. In fact, manufacturers' data sheets show only one value for attenuation.

However, if we take into consideration the existence of modes in a multimode optical fiber, we will arrive at a conclusion other than the one noted above because different modes travel significantly different distances within the same fiber. In other words, even though the length of a fiber is the same for all modes, the traveling distance is quite different for the various modes. Since high-order modes travel significantly longer distances within the same core than their low-order counterparts, they experience much more absorption, scattering, and microbending events, which results in greater losses for such modes. The result, then, is that high-order modes disappear much faster than low-order modes. Therefore, our conclusion is this: The longer the fiber, the fewer the number of modes arriving at the receiving end.

For a very long fiber, this process reaches the steady-state condition called equilibrium-mode distribution, meaning that only low-order modes continue to propagate inside a long optical fiber. But these modes experience less loss than their high-order counterparts simply because they travel a shorter distance within the fiber. Therefore, for a short piece of optical fiber, where all modes exist, we will measure the higher level of loss, while for a longer 
piece of fiber, where only low-order modes are left, we will measure the lower level of loss. In other words, for multimode fiber, attenuation should decrease with an increase in fiber length.

If we compare graphs of attenuation versus fiber length for MMF and for singlemode fiber (SMF), we should see that for an $S M F$, attenuation should be constant whereas for $M M F$, attenuation should decrease over the fiber length. We need to understand that this experiment proves the existence of modes in optical fiber and that there are no direct measurements that can prove this existence because we can't look inside a fiber.

In addition to the above reasoning, the students have to describe in their laboratory reports the industry approaches to these measurements. They have to find out, for instance, that there is a method of measuring attenuation defined in a fiber-optic test-procedure (FOTP) standard accepted by the Electronic Industry Alliance (EIA) and the Telecommunications Industry Association (TIA). They also have to analyze the difference between their measurements and those performed in accordance with this standard.

We take a similar approach we assign our students the task of explaining how attenuation in optical fiber depends on the wavelength of a light source. Here the students have to use the graph "Attenuation vs. wavelength" given in a manufacturer's data sheet. They have to analyze this graph ("the longer the wavelength, the smaller the attenuation") and explain that it is the absorption that determines the course of this graph.

\section{Discussion of fiber-bandwidth theory}

Here we want to investigate the bandwidth (transmission capacity) of an optical fiber. The objective is to develop a scientific understanding of this performance characteristic of the optical fiber given in the manufacturer's data sheet during a classroom discussion of fiber-bandwidth theory.

Bandwidth is the second basic characteristic of a transmission link. Optical fiber has become the dominant transmission medium simply because it has the largest bandwidth among all other existing media. This is the major advantage of an optical fiber over a microwave link, a coaxial cable or a copper wire. The theoretical limit of the bandwidth of optical fiber is about $50 \mathrm{Tbit} / \mathrm{s}$; however, a close look reveals a number of obstacles that restrict this bandwidth to a much lower number. The study of bandwidth inevitably brings students to a discussion of dispersion.

Intermodal and chromatic dispersions are discussed based on the physical processes of light propagation within an optical fiber. This discussion is followed by a consideration of how these dispersions affect the bandwidth (bit rate) of an optical fiber.

The study of this topic is concluded by a review of a data sheet of an optical fiber. For our class discussion, the specifications sheet from Plasma Optical Fibre Company (given in the textbook ${ }^{1}$ ) is used; as homework, students are assigned to review the latest data sheets from different manufacturers (Corning, Lucent Technologies, Pirelli, SpecTran, and others). The formats and examples of such specifications sheets are presented in the course textbook ${ }^{1}$.

This is what we expect students to learn as a result of our consideration of the bandwidth theory of a multimode optical fiber given in a manufacturer's data sheet:

The manufacturer specifies bandwidth as a set of numbers at different wavelengths. These numbers are given even without units. For example, the data sheet says that the bandwidth of MMF at 850/1300 is equal to 200/400. The problem is to analyze these data.

First, since bandwidth is restricted by intermodal and chromatic dispersions and since intermodal dispersion puts severe limitations on bandwidth, we must conclude that this specification is determined by intermodal dispersion. Here we need to recall that intermodal dispersion is the phenomenon leading to the spread of the output pulse. This widening stems from the fact that light inside a fiber breaks down into separate discrete beams called modes. These beams travel at different angles with respect to the fiber's centerline; therefore, they arrive at the receiver end at different times. The output pulse is composed of the small pulses delivered by the individual modes. Thus, the front edge of the output pulse is determined by the fastest mode, while its rear edge is determined by the slowest mode. This is why the output pulse is spread in contrast to the input pulse.

Secondly, bandwidth, as already noted, is specified in a data sheet without mention of its units. This raises the question of how manufacturers measure fiber bandwidth. We find out that manufacturers specify bandwidthlength product rather than pure bandwidth, which means that they use $\mathrm{MHz}-\mathrm{km}$ rather than $\mathrm{MHz}$.

Third, to understand why manufacturers specify bandwidth as two numbers, we need to consider what essentially changes in optical fiber with a change in operating wavelength. The first-and the main-reason for the change is that the number of modes, $N$, depends on the wavelength. Indeed, this number is given as

$$
N=V^{2} / 2 \text { or } N=V^{2} / 4
$$


where

$$
V=(\pi d N A) / \lambda
$$

Here $d$ is the core diameter, NA is the numerical aperture, and $\lambda$ is the operating wavelength [1]. From these calculations we come to the conclusion that the shorter the wavelength, the greater the number of modes within a fiber and the wider the pulse spread caused by intermodal dispersion and, therefore, the lower the bandwidth.

The second reason fro the change is that chromatic dispersion depends on the wavelength. In fact, pulse spreading caused by chromatic dispersion can be calculated as follows:

$$
\Delta t_{\text {chrom }}=D(\lambda) \Delta \lambda L
$$

where $D(\lambda)$ is the chromatic-dispersion parameter measured in picoseconds (ps) per nanometer ( $\mathrm{nm}$ ) times

kilometer $(\mathrm{km}), L$ is the transmission length in $\mathrm{km}$, and $\Delta \lambda$ is the spectral width of the light source in $\mathrm{nm}$. Given the spectral width of the light source and the transmission length, the chromatic-dispersion parameter becomes the critical characteristic of an optical fiber that determines chromatic dispersion. Manufacturers specify the chromatic-dispersion parameter of optical fibers either by giving its value or by the formula

$$
D(\lambda)=\left(S_{0} / 4\right) \lambda\left[1-\left(\lambda_{0} / \lambda\right)^{4}\right]
$$

where $S_{0}$ is the zero-dispersion slope in $\mathrm{ps} /\left(\mathrm{nm}^{2}-\mathrm{km}\right), \lambda_{0}$ is the zero-dispersion wavelength, and $\lambda$ is the operating wavelength. Thus, we can see why the bandwidth depends on the wavelength: Bandwidth depends on pulse spread; pulse spread, in turn, depends on chromatic dispersion; lastly, chromatic dispersion depends on the wavelength.

This is the kind of discussion students have to present in their term papers; they also have to be ready to demonstrate such knowledge on examinations.

The above examples show how we arrange virtual collaboration with industry. Our experience shows that such an approach can be very successful when teaching fiber-optic communications at the senior level in the undergraduate telecommunications engineering technology program.

\section{Summary}

The main challenge in teaching a fiber-optic communications course-where the subject matter is the physical processes of components and systems - in engineering technology programs is the limited scientific background of the students. Also, it is important for engineering technology programs to simulate collaboration with industry so that students gain "real-world" experience even before they start their professional career.

To accomplish this, we propose adoption of a program we call virtual collaboration, in which the teaching process simulates the true-to-life working environment and the teaching material relies heavily on real technical documentation, such as specifications sheets and technical notes.

Such an approach would entail, for example, the use of the specifications sheets of a multimode optical fiber (MMF). Through discussion of MMF attenuation, an understanding of the mechanisms of losses within an MMF should convince the students that (a) attenuation should be independent of fiber length, although the existence of modes will result in decreasing attenuation with length; and (b) attenuation depends on the wavelength of the transmitted signal. To verify these facts, the students should be able to develop the test procedure and perform measurements in the laboratory.

Bandwidth (transmission capacity) is the second basic characteristic of a transmission link. Understanding the theory of dispersion allows students to comprehend why real MMF bandwidth is restricted to $200 / 400 \mathrm{MHz}-\mathrm{km}$ at $850 / 1300 \mathrm{~nm}$, as given in a typical data sheet. By working out simple formulas involving the relationship of the number of modes to the operating wavelength and dependence of chromatic-dispersion parameter on the wavelength, students are able to discern why manufacturers specify bandwidth at each $\lambda$.

\section{References:}

1. Djafar K. Mynbaev and Lowell L. Scheiner, Fiber-Optic Communications Technology (Prentice Hall, 2001).

2. Djafar K. Mynbaev, Laboratory Manual for course TC 700 - Fiber-Optic Communications (New York City College of Technology, Brooklyn, NY, 2003). 